\title{
Exomorfología y anatomía de órganos vegetativos aéreos en especies de Flourensia DC. (Asteraceae) con importancia fitoquímica
}

\author{
Natalia Delbón ${ }^{1,2,3}$, María Teresa Cosa² y Gabriel Bernardello
}

Recebido em 21/06/2011. Aceito em 18/10/2011

\begin{abstract}
RESUMEN
(Exomorfología y Anatomía de órganos vegetativos en especies de Flourensia DC. (Asteraceae) con importancia fotoquímica). El género Flourensia DC. es americano y comprende 32 especies. Son arbustos resinosos utilizados en medicina popular; poseen un potencial valor económico, por lo que, en algunas especies representantes, se han caracterizado sus compuestos químicos y determinado algunos posibles usos. En el presente trabajo, se estudió la exomorfología y anatomía de órganos vegetativos de F. hirta S. F. Blake, F. leptopoda S. F. Blake, F. niederleinii S. F. Blake y F. tortuosa Griseb., especies endémicas del centro de Argentina. Se observó que todas son similares en cuanto a la anatomía de hoja y tallo. En cuanto al estudio de la epidermis foliar, se concluye que es posible diferenciar las especies teniendo en cuenta la frecuencia estomática y la presencia de tricomas. Finalmente, las diferencias más notables se encontraron en la exomorfología foliar ya que se observó una gran variación en cuanto a la forma de las hojas y tamaño de las láminas y pecíolos. Con respecto a las estructuras secretoras, se encontraron tricomas y conductos secretores esquizógenos en las hojas y los tallos de todas las especies, las cuales serían responsables de la producción y secreción de las resinas.
\end{abstract}

Palabras clave: estructuras secretoras, hoja, tallo

\begin{abstract}
(Exomorphology and anatomy of vegetative organs in Flourensia species (Asteraceae) with phytochemical importance). The genus Flourensia DC. is American and comprises 32 species. They are resiniferous shrubs used in folk medicine. As they have potential economic value, its chemical compounds have been characterized and some possible uses identified. In this work, the exomorphology and anatomy of vegetative organs of F. hirta S. F. Blake, F. leptopoda S. F. Blake, F. niederleinii S. F. Blake and F. tortuosa Griseb., endemic species from central Argentina, have been studied. All species are similar in leaf and stem anatomy. Regarding the epidermis, it is possible to differentiate the species according to the stomatal frequency and the presence of trichomes. Finally, the most remarkable differences were found in leaf exomorphology, since there was a large variation in shape and size of blades and petioles. Concerning the secretory structures, trichomes and secretory ducts were found in the leaves and stems of all species, which would be responsible for the production and secretion of the resins.
\end{abstract}

Key words: leaf, secretory structures, stem

\section{Introducción}

En Asteraceae es común la presencia de estructuras secretoras entre las que se destacan conductos, cavidades y tricomas de diferentes tipos. Sus secreciones pueden contener diferentes compuestos químicos, entre ellos terpenos, presentes en aceites esenciales y resinas, carbohidratos $\mathrm{u}$ otras sustancias, lo que les confiere un gran valor fitoquímico (Fahn 1979; Evert 2006).
El género Flourensia DC. (Asteraceae, Asteroideae, Tribu Heliantheae) es exclusivamente americano y comprende 32 especies (Dillon 1984; Ariza Espinar 2000; Sáenz 2000). En Argentina crecen 12 de ellas, en tanto que en la zona central del país habitan 6; su importancia se debe a que todas son endémicas de esa zona y presentan distribución restringida. Habitan en zonas serranas, en ambientes caracterizados por un clima semiárido y suelos empobrecidos (Luti 1979). Son arbustos con capítulos radiados de flores amarillas, abun-

\footnotetext{
1 Universidad Nacional de Córdoba, CONICET, Córdoba, Argentina

2 Universidad Nacional de Córdoba, Facultad de Ciencias Exactas, Físicas y Naturales, Instituto Multidisciplinario de Biología Vegetal, CONICET, Córdoba, Argentina

3 Autor para correspondência: natalia_delbon@hotmail.com
} 
dantes y muy vistosas. Presentan un característico aspecto lustroso debido al exudado resinoso de sus hojas y ramas (Ariza Espinar 2000).

Son reconocidas en medicina popular ya que se las utiliza para aliviar diversas enfermedades gastrointestinales, como purgante, expectorante y antirreumática; además algunas especies son usadas como incienso, aromáticas y tintóreas (Zardini 1984; Mata et al. 2003; Barbosa et al. 2006; Trillo et al. 2007; Zavala et al. 2010).

Debido a que sus resinas poseen un potencial valor económico, numerosas investigaciones han sido dirigidas a caracterizar sus compuestos químicos y determinar los posibles usos. Se identificaron los componentes del aceite esencial de F. oolepis S. F. Blake (Priotti et al. 1997; García et al. 2007; Díaz Nepal et al. 2009) y se comprobó su efecto bactericida (Carrizo Flores et al. 2005; Vaca Ruiz et al. 2006; Joray et al. 2011), insecticida (García et al. 2007), antifúngico (Carpinella et al. 2010) y protector contra úlceras gástricas (Donadel et al. 2005). Además, se estudiaron los compuestos químicos en F. campestris Griseb., F. riparia Griseb., F. fiebrigii S. F. Blake (Uriburu et al. 2004, 2005, 2007), F. blakeana Dillon (Pacciaroni et al. 2009), F. heterolepis S. F. Blake (Bohlmann \& Jakupovic 1979) y F. cernua DC. (Rao et al. 1970; Estell et al. 1996; Tellez et al. 1997). En esta última, se comprobó su acción antifúngica (Gamboa-Alvarado et al. 2003), fitotóxica (Mata et al. 2003), bactericida (MolinaSalinas et al. 2006) e insecticida (Gerrero-Rodriguez et al. 2007; Tellez et al. 2001). Por su parte, Jasso de Rodríguez et al. (2007) reconocieron el efecto antifúngico de los extractos de F. microphylla (Gray) S. F. Blake, F. cernua y F. retinophylla S. F. Blake.

En contraste, poco es lo que se conoce acerca de la morfoanatomia de las especies de Flourensia y de las estructuras responsables de la producción y secreción de las resinas. Al respecto, en investigaciones anteriores, analizamos la anatomía de los órganos vegetativos de F. campestris y $F$. oolepis, (Delbón et al. 2007a) destacando la presencia de tricomas glandulares y conductos secretores esquizógenos distribuidos en todos los órganos vegetativos, ambas estructuras serían las encargadas de producir las resinas. En el presente trabajo se propone continuar el estudio de especies de este género, analizando la exomorfología y anatomía de órganos vegetativos aéreos de $F$. hirta, F. leptopoda, $F$. niederleinii y $F$. tortuosa para aportar información inédita sobre estos arbustos endémicos y contribuir a su diferenciación sistemática.

\section{Materiales y métodos}

Las especies analizadas proceden de Argentina. El material de herbario correspondiente se encuentra depositado en el Museo Botánico de Córdoba (CORD).

Flourensia hirta S. F. Blake. ARGENTINA. La Rioja: Campana, S 28 33'; W 67 37', 1625 msm, 16-II-2009, Delbón 3.
F. leptopoda S. F. Blake. ARGENTINA. La Rioja: Ulapes, S 31 34'; W 66ํ14', 734 msm, 16-II-2009, Delbón 4.

F. niederleinii S. F. Blake. ARGENTINA. La Rioja: Sanagasta, RN 75, km 45. S $29^{\circ} 11^{\prime}$; W 67 0,3', 1212 msm, 16-II-2009, Delbón 1.

F. tortuosa Griseb. ARGENTINA. Catamarca: Andalgalá, S 27 32'; W 66 17', 1246 msm, 16-II-2009, Delbón 2.

De cada población se tomaron muestras de cuatro individuos elegidos al azar, las cuales fueron fijadas en FAA. Las hojas maduras fueron recolectadas en la zona apical de las ramas laterales. Para los estudio de la lámina foliar se utilizó la región del tercio medio de la misma; para el estudio del pecíolo se trabajó con su parte media y proximal. El estudio del tallo fue realizado en primer y segundo entrenudo inmediatamente por debajo de la yema apical.

Se estudió la epidermis foliar para lo cual se seleccionaron dos hojas de cada individuo y se realizaron extendidos de ambas caras siguiendo la técnica de raspado de Metcalfe (D'Ambrogio de Argüeso 1986). Para el estudio de los tricomas se utilizó la clasificación y terminología propuesta por Ramayya (1962) para la familia Asteraceae.

Se analizó la anatomía de la lámina foliar ello se realizaron preparados permanentes de cortes transversales. El material conservado en FAA se deshidrató en una serie de alcohol etílico y xilol y se lo incluyó en histoplast, los cortes microtómicos de $10 \mu \mathrm{m}$. se colorearon con azul astral - fucsina básica y finalmente se montaron con bálsamo de Canadá (Kraus et al. 1998). Además, se realizaron preparados temporarios de cortes transversales por pecíolo y tallo, los cuales se tiñeron con azul astral - fucsina básica y se montaron con glicerina $50 \%$.

Se estudiaron las características cuantitativas exomorfológicas de las hojas para lo cual se utilizaron cinco hojas de cada individuo; se midió el lago, ancho y espesor de la lámina y del pecíolo y el espesor de la vana media. Con el programa Leaf Area Measurement (Askew 2003) se calculó el área foliar total (lámina y pecíolo).

Por último, se realizó un estudio cuantitativo de la epidermis foliar en vista superficial, para ello se eligieron al azar tres campos de cada extendido y se cuantificaron las células epidérmicas propiamente dichas y los estomas. Se determinó el índice estomático para ambas epidermis, en cada especie, con la fórmula:

I.E.= (frecuencia de estomas / frecuencia de estomas + frecuencia de células epidérmicas) x 100 (Stace 1965).

Todas las variables fueron analizadas estadísticamente con el programa InfoStat (Di Rienzo et al. 2009). Se calcularon las medias y desvíos estándares y luego se realizaron Análisis de Varianza y Test de Tukey con el objetivo de determinar si existen diferencias significativas entre las especies (Scheffé 1959; Sokal \& Rohlf 1981).

\section{Resultados}

Las especies analizadas presentan hojas alternas, verde oscuro, muy brillantes y resinosas (Fig. 1 - 4). Son peciola- 
das, excepto en $F$. hirta en la que el pecíolo es muy breve (Fig. 1). La forma de las hojas varía considerablemente entre las especies, siendo angostas y lanceoladas en F. hirta (Fig. 1), rómbico - ovaladas y con margen aserrado en $F$. leptopoda (Fig. 2), aovadas o elípticas en F. niederleinii (Fig. 3) y de aovadas a lanceoladas en F. totuosa (Fig. 4).

Las observaciones anatómicas de las hojas muestran que, en la epidermis, los estomas son de tipo anomocíticos, se hallan rodeados por 4 a 6 células epidérmicas propiamente dichas (Fig. 5 - 8) y se diferencian en ambas caras foliares. Las células epidérmicas propiamente dichas son irregulares, las paredes anticlinales son sinuosas en F. hirta (Fig. 5) y ligeramente sinuosas en las otras 3 especies.

Se presentan tres tipos de tricomas:

- Filiforme Simple: Pie simple, unicelular. Cuerpo pluricelular, uniseriado, entero, formado por 3 a 6 células isodiamétricas, finalizando en una célula apical alargada con contenido algo oscuro, opaco, dado posiblemente por la presencia de sustancias pécticas (Fig. 9). Son frecuentes en todas las especies, en ambas caras de las hojas y se distribuyen siguiendo la venación.

- Cónico Simple: pluricelular uniseriado, entero de forma cónica y con su extremo ahusado, formado por 2 ó 3 células de diferente longitud (Fig. 10 y 11). Son escasos en F. niederleinii y F. leptopoda y frecuentes en F. tortuosa y F. hirta; en ésta última, son de mayor tamaño y abundantes en los bordes de las hojas.

- Glandular Vesicular Biseriado - subtipo $\beta$ : pie compuesto, bicelular biseriado. Cuerpo biseriado, entero, de forma oblonga, compuesto por cuatro o cinco pares de células de contenido denso (Fig. 12 y 13). Abundantes en todas las especies, en ambas caras foliares y en especial siguiendo la venación.

La lámina foliar en corte transversal muestra que la epidermis es unistratificada, con cutícula gruesa, células epidérmicas propiamente dichas isodiamétricas y estomas al mismo nivel (Fig. 14 - 16). El mesofilo tiene estructura isolateral, Es notablemente laxo en F. leptopoda y F. hirta (Fig. 14) y más compacto en las otras especies (Fig. $15 \mathrm{y}$ 16). El paránquima esponjoso es incipiente mientras que el parénquima en empalizada se encuentra muy desarrollado, en algunos sectores, éste se desorganiza por la presencia de grandes espacios intercelulares y cámaras subestomáticas. El sistema vascular está representado por hacecillos colaterales cerrados. Los más pequeños constan de unos pocos elementos del xilema y floema, rodeados unas pocas células parenquimáticas de la vaina. Los haces mayores además se encuentran acompañados de grupos de fibras y se hallan rodeados en una vaina con mayor desarrollo, la cual se prolonga hacia ambas epidermis, constituyendo las extensiones de la vaina (Fig. 15). El borde foliar sigue la misma anatomía general y culmina en su extremo con un grupo de células parenquimáticas, en esta zona las células epidérmicas presentan una cutícula notablemente mas gruesa (Fig. 16).
La vena media consta de un sólo haz central en $F$. hirta (Fig. 17) y F. leptopoda (Fig. 18), tres en F. niederleinii y tres o cinco en F. tortuosa (Fig. 19); algunos hacecillos pueden estar acompañados por fibras.

En el pecíolo, la epidermis es unistratificada y la cutícula es gruesa, se encuentran abundantes tricomas en especial del tipo glandular. Por debajo de la epidermis hay 2 ó 3 estratos de colénquima angular compacto $y$, por dentro, unos 6 estratos de parénquima. Los hacecillos vasculares son colaterales cerrados y en general están acompañados por fibras. En F. hirta (Fig. 20) hay 3 a 5 hacecillos y conductos secretores sólo hacia el hipofilo. En F. leptopoda (Fig. 21) y F. niederleinii hay 3 a 7 hacecillos y los conductos secretores se encuentran hacia ambas caras. F. tortuosa presenta 5 a 9 hacecillos y los conductos secretores son de mayor diámetro y se diferencian hacia el hipofilo, con poca frecuencia aparecen algunos hacia el epifilo.

Los tallos jóvenes son verde oscuro, pubescentes y resinosos; con la formación de la corteza en el crecimiento secundario se vuelven color castaño oscuro.

Son circulares en corte transversal. La epidermis es unistratificada con cutícula engrosada y los tricomas son abundantes en las partes jóvenes en especial los glandulares. Por debajo de la epidermis se encuentran 4 a 6 estratos de colénquima angular y 5 a 7 de parénquima. El tejido vascular se dispone inicialmente formando una eustela. Los hacecillos son colaterales abiertos y están separados por un parénquima interfascicular poco conspicuo, los haces mayores presentan un casquete notable de fibras por fuera del floema y en menor cantidad hacia la médula (Fig. 22).

El crecimiento en espesor comienza en estados tempranos del desarrollo de los tallos. El felógeno tiene su origen en el primer estrato de células corticales, inmediatamente por debajo de la epidermis (Fig. 23).

En las cuatro especies estudiadas se encuentran conductos secretores, siempre relacionados al sistema vascular. En la lámina forman parte de la vaina del haz y sus extensiones (Fig. 14 - 16). En la vena media (Fig. 17 - 19), pecíolo (Fig. 20 y 21) y tallo (Fig. 22 y 23) se observan conductos secretores en el parénquima, en los estratos próximos a los hacecillos vasculares. Además, unos pocos conductos pueden aparecer en la médula de los tallos. Analizando los cortes longitudinales y transversales del apice caulinar (Fig. 24) se puede observar que los conductos son espacios largos que recorren longitudinalmente al órgano y están limitados por un epitelio unistratificado de células secretoras de contenido denso. Son de origen esquizógeno ya que, en estadios muy jóvenes de los pimordios foliares, éstos se forman por separación de las paredes de las células vecinas sin que éstas se desintegren (Fig. 25).

\section{Caracteres cuantitativos}

El tamaño foliar varía considerablemente entre las especies (Tab. 1). Los análisis de varianza (con $p \leq 0,05$ y 3 grado de libertad) muestran que existen diferencias significativas en todas las variables analizadas. Los test de Tukey indican 

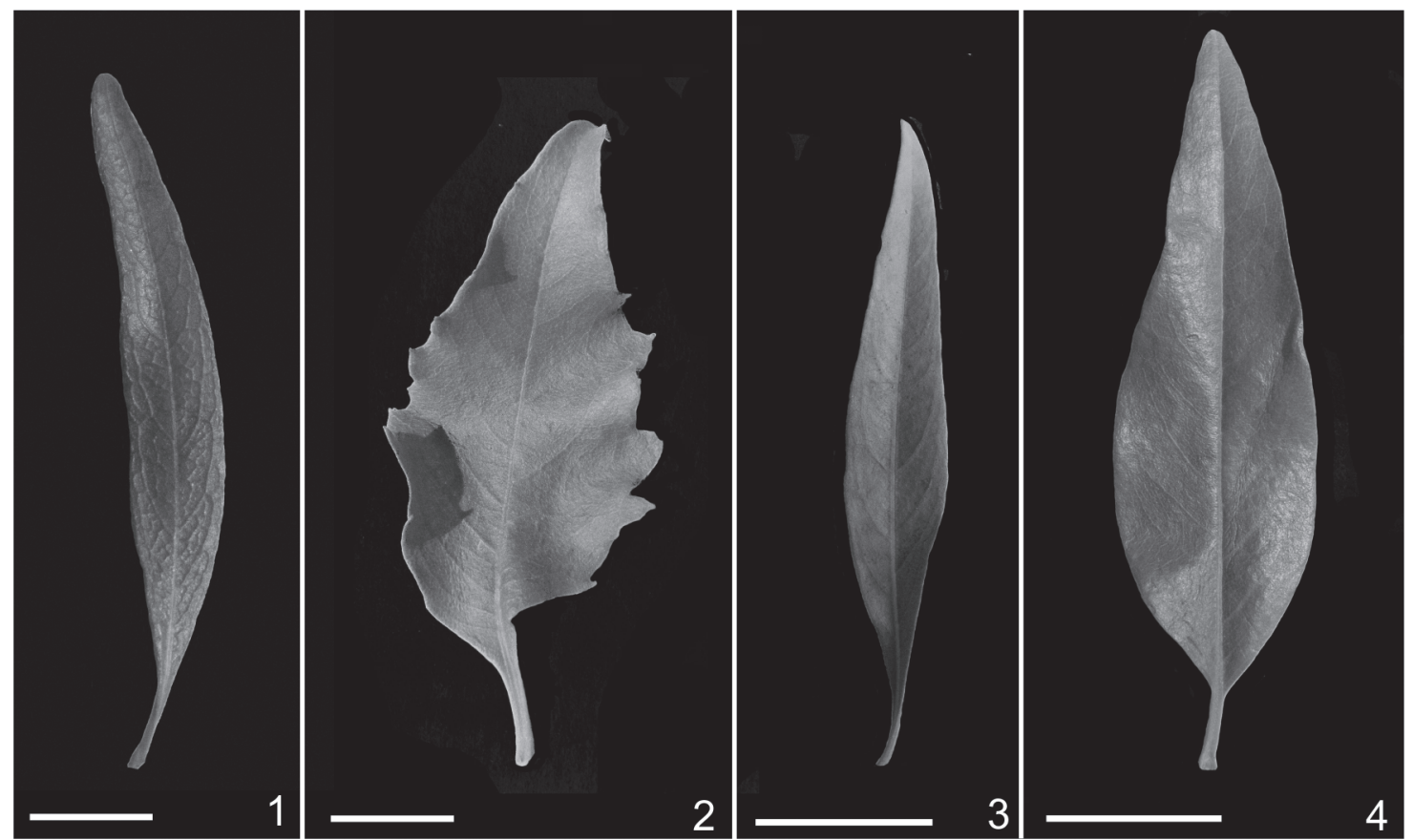

Figura 1-4. exomorfología foliar de especies de Flourensia DC. (Asteraceae). 1, F. hirta. 2, F. leptopoda. 3, F. niederleinii. 4, F. tortuosa. Escala: 1 y $2=10 \mathrm{~mm} .3$ y $4=20 \mathrm{~mm}$.

entre que especies hay diferencias. Cabe destacar que F. tortuosa presenta los mayores valores en todas las mediciones, en contraste, en $F$. hirta se observan los menores valores en la mayoría de las variables.

Análisis de las epidermis foliares (Tab. 2), se muestran las medias y desvíos estándares de las variables frecuencias de estomas y de células epidérmicas propiamente dichas, para ambas epidermis foliares de cada especie. Se realizaron comparaciones entre las epidermis foliares de cada especie para las variables mencionadas. Los resultados del análisis de la Varianza muestran que la frecuencia de estomas es significativamente diferente entre las epidermis de F. tortuosa, F. niederleinii y F. leptopoda, siendo en todos los casos mayor en el hipofilo; en F. hirta no se encontraron diferencias significativas. La frecuencia de células epidérmicas sólo es significativamente diferente entre las epidermis foliares de F. tortuosa, siendo menor en el epifilo.

Luego se comparó la frecuencia de estomas del hipofilo y epifilo de todas las especies. Los Análisis de Varianza (con $p \leq 0,05$ y 3 grados de libertad) indican que existen diferencias significativas en ambas variables. Los resultados del test de Tukey (Tab. 3) muestran que teniendo en cuenta las frecuencias estomáticas de los hipofilos, F. tortuosa y $F$. hirta forman un grupo con valores bajos mientras que $F$. niederleinii y F. leptopoda forman otro grupo con valores altos. Por otro lado, teniendo en cuenta la frecuencia estomática de los epifilos, las especies se distribuyen en tres grupos: $F$. tortuosa con un valor muy bajo, F. hirta y F. niederleinii juntas, con valores intermedios y finalmente $F$. leptopoda con un valor comparativamente muy alto.

\section{Discusión y conclusiones}

Las especies de Flourensia aquí estudiadas presentan una gran similitud en la morfoanatomía de sus órganos vegetativos, así como a las ya estudiadas previamente $F$. campestris y F. oolepis (Delbón et al. 2007a). Se encontraron abundantes estomas anomocíticos en ambas caras foliares, lo cual se considera ventajoso en ambientes xéricos, ya que permitirían una mayor eficiencia en el intercambio gaseoso, en poco tiempo (Fahn 1990; Fahn \& Cutler 1992). En cuanto a la anatomía de las hojas, cabe destacar que en todas las especies el parénquima en empalizada presenta un gran desarrollo, a expensas del esponjoso que es casi indiferenciable, esto sería favorable ya que incrementaría la actividad fotosintética (Fahn 1990).

Las diferencias anatómicas más relevantes entre las especies se relacionan con el sistema vascular. F. hirta y $F$. leptopoda presentan menor número de hacecillos vasculares en la vena media y en el pecíolo, en comparación con las restantes especies, incluida F. campestris y F. oolepis; esto podría ser considerado como una consecuencia de la reducción del tamaño de las hojas.

Asimismo, es posible diferenciar las especies teniendo en cuenta una característica cuantitativa epidérmica de gran valor taxonómico como lo es la frecuencia estomática. Los análisis estadísticos realizados muestran que existen diferencias significativas entre las especies, siendo F. leptopoda la especie que presenta mayor frecuencia en ambas caras foliares. Por otro lado, en todas las especies, excepto F. hirta, el hipofilo presenta mayor frecuencia estomática que el epifilo. Estos resultados están en concordancia con 


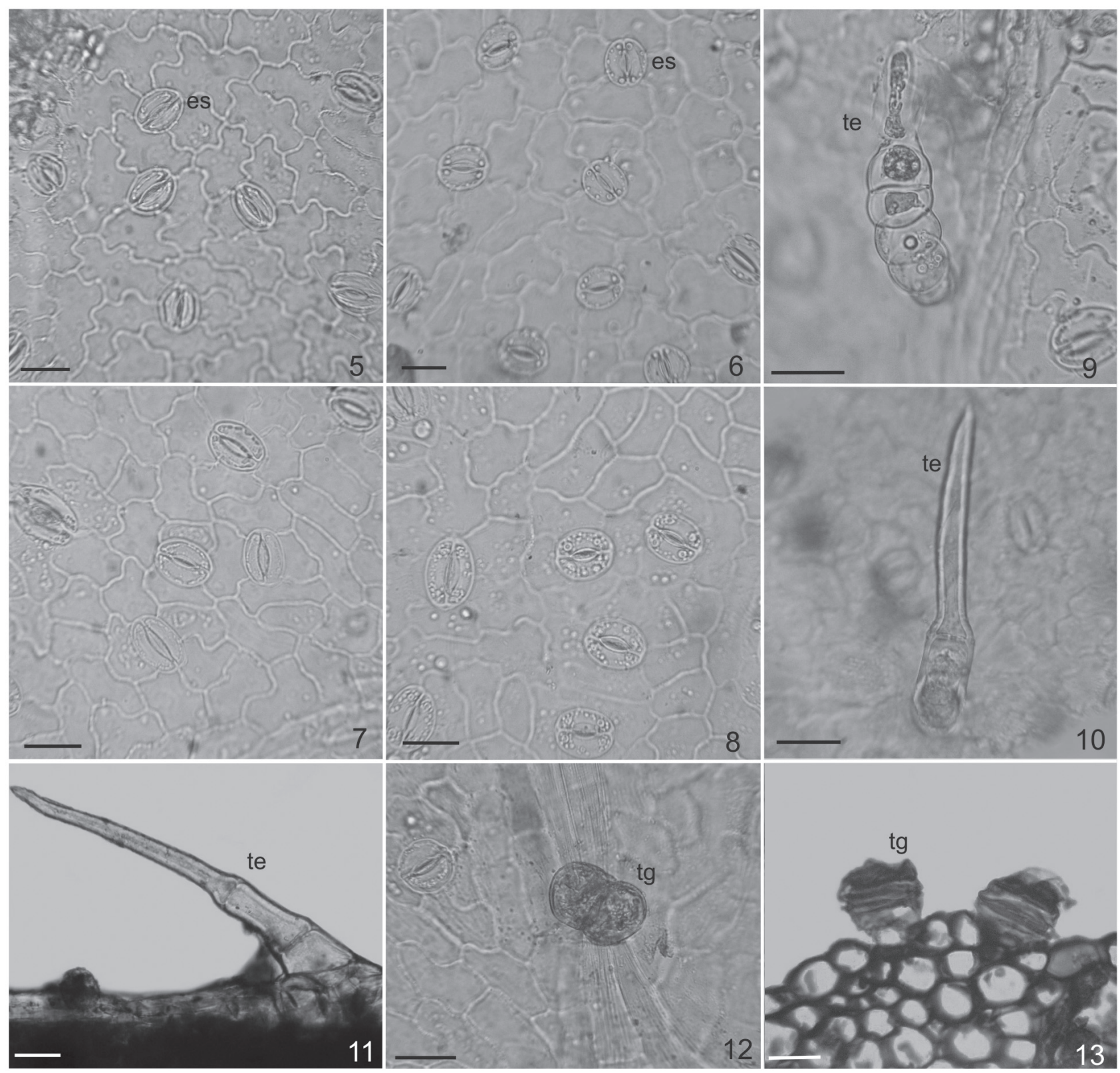

Figura 5-13. epidermis de especies de Flourensia DC. (Asteraceae). 5 - 8 epidermis en vista superficial. 9 - 13 tricomas. 5, 9 y 11, F. hirta. 6, F. leptopoda. 7 y 13, F. niederleinii. 8,10 y 12, F. tortuosa. Escala: $30 \mu \mathrm{m}$. Abreviaturas: es: estoma, te: tricoma eglandular, tg: tricoma glandular.

los anteriormente publicados (Delbón et al. 2007b), en $F$. campestris la frecuencia de estomas fue de $170,82 \pm 27,18$ en el epifilo y de 198,03 $\pm 35,42$ en el hipofilo y en $F$. oolepis de $149,64 \pm 22,3$ en el epifilo y de $198,03 \pm 35,42$ en el hipofilo.

Finalmente, las diferencias más notables entre las especies se encontraron en la exomorfología foliar ya que se observó una gran variación en cuanto a la forma de las hojas y tamaño de las láminas y pecíolos. F. tortuosa es la especie que presenta los valores mayores en todas las mediciones realizadas, en contraste, se observa en $F$. hirta y $F$. leptopoda los valores menores. Según varios autores, la reducción del tamaño de las hojas en especies de ambientes áridos podría ser considerada una importante adaptación, ya que dismi- nuiría la pérdida de agua por transpiración (Fahn 1990; Fahn \& Cutler 1992; Dickison 2000); teniendo en cuenta este hecho, F. hirta y F. leptopoda podrían ser consideradas las especies mejor adaptadas al ambiente en que viven. Resaltamos en este punto, la importancia del tamaño y la forma de las hojas como caracteres taxonómicos fundamentales para la identificación de las especies.

Con respecto a las estructuras secretoras, se encontraron tres tipos de tricomas en las especies aquí estudiadas, dos eglandulares y uno glandular. Estas observaciones no coinciden totalmente con las realizadas en F. campestris y F. oolepis ya que en éstas no se hallaron tricomas egladulares del tipo cono simple. (Delbón et al. 2007b). Los tricomas glandulares también 

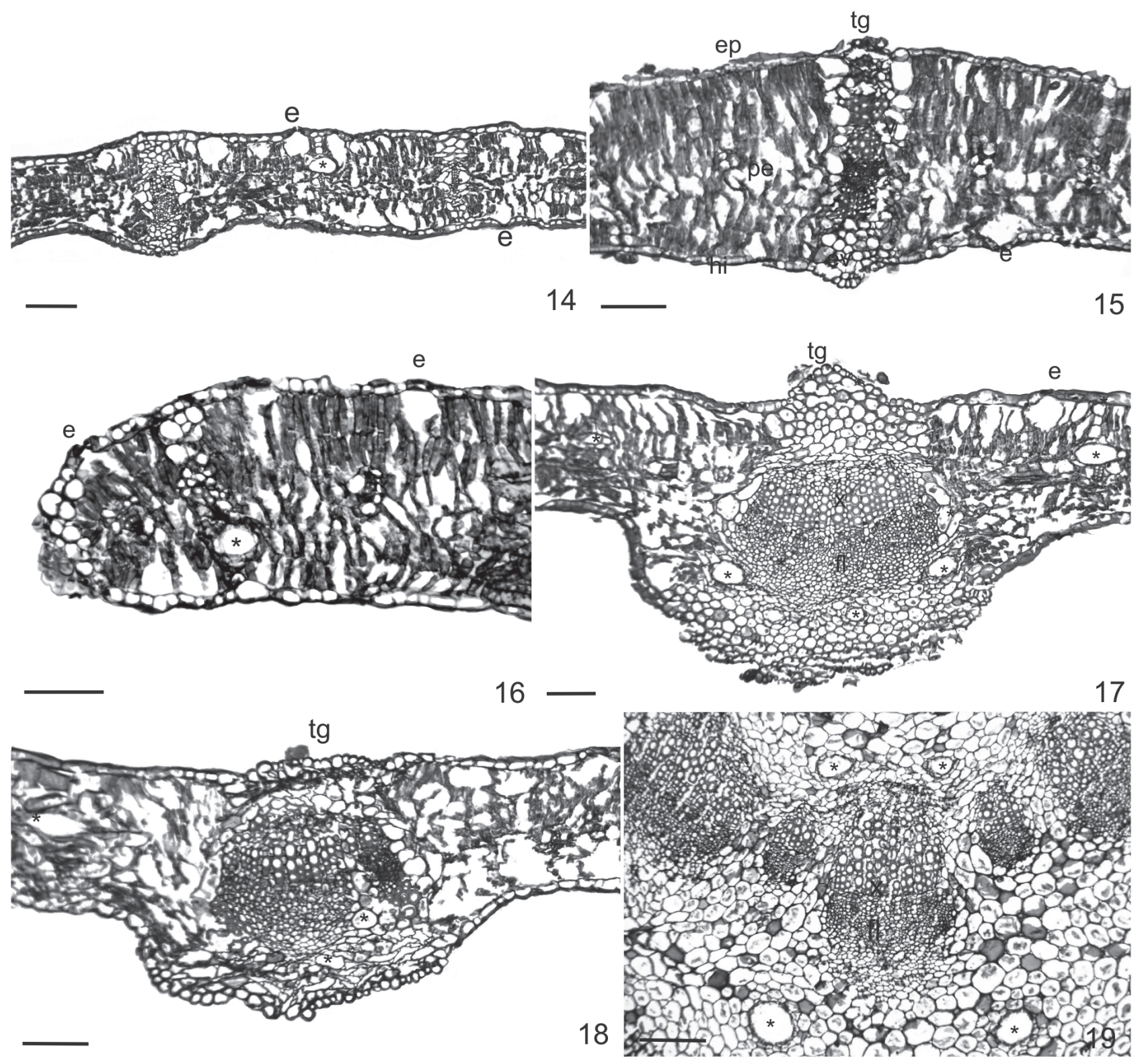

Figura 14-19. Corte transversal por hoja de Flourensia DC. (Asteraceae). 14, vista general del mesofilo de $F$. hirta. 15, detalle de hacecillo de conducción de $F$. tortuosa. 16, borde foliar de F. niederleinii. 17 y 18, nervio medio de F. hirta y F. leptopoda. 19, detalle de hacecillos de conducción en vena media de F. tortuosa. Escala: $100 \mu \mathrm{m}$. Abreviaturas: e: estoma, es: parénquima esponjoso, ep: epifilo, ev: estenciones de la vaina, fl: floema, hi: hipofilo, tg: tricoma glandular, v: vaina, x: xilema, ${ }^{*}$ conductos secretores.

ha sido descritos en Siegesbeckia orientalis L. (Aguilera et al. 2004), especie perteneciente a la misma tribu (Heliantheae), en Stevia rebaudiana Bert. (Monteiro et al. 2001) de la tribu Eupatorieae, filogenéticamete cercana a Heliantheae (Funk et al. 2009) y en Baccharis coridifolia DC., tribu Astereae (Budel \& Duarte 2007); en estas dos últimas especies, se observó que los tricomas reaccionaron positivamente a los test de compuestos lipofílicos y fenólicos, confirmando su carácter secretor. Desde el punto de vista ecológico, la resina secretada por los tricomas glandulares podría jugar un rol importante en la reducción de la pérdida de agua por transpiración, por lo que esta característica es considerada como un adaptación a ambientes áridos (Fahn \& Cutler 1992).
Con respecto a los conductos secretores, debe señalarse que se encontraron en todos los órganos vegetativos de todas las especies y siempre asociados al sistema vascular; son de origen esquizógeno y presentan un epitelio secretor unistratificado. Estos resultados concuerdan con los encontrados anteriormente en F. campestris y F. oolepis (Delbón et al. 2007a), así como en otros géneros de Asteraceae (Castro et al. 1997; Melo de Pinna \& Menezes 2002; Simon et al. 2002; Lapp et al. 2004; Molares et al. 2009). Aguilera et al. (2004) observaron que las secreciones de los conductos reaccionaron positivamente a los tests de compuestos fenólicos y alcaloides. La secreción interna de terpenos y otras sustancias ayudarían a proteger la planta de herbívoros 

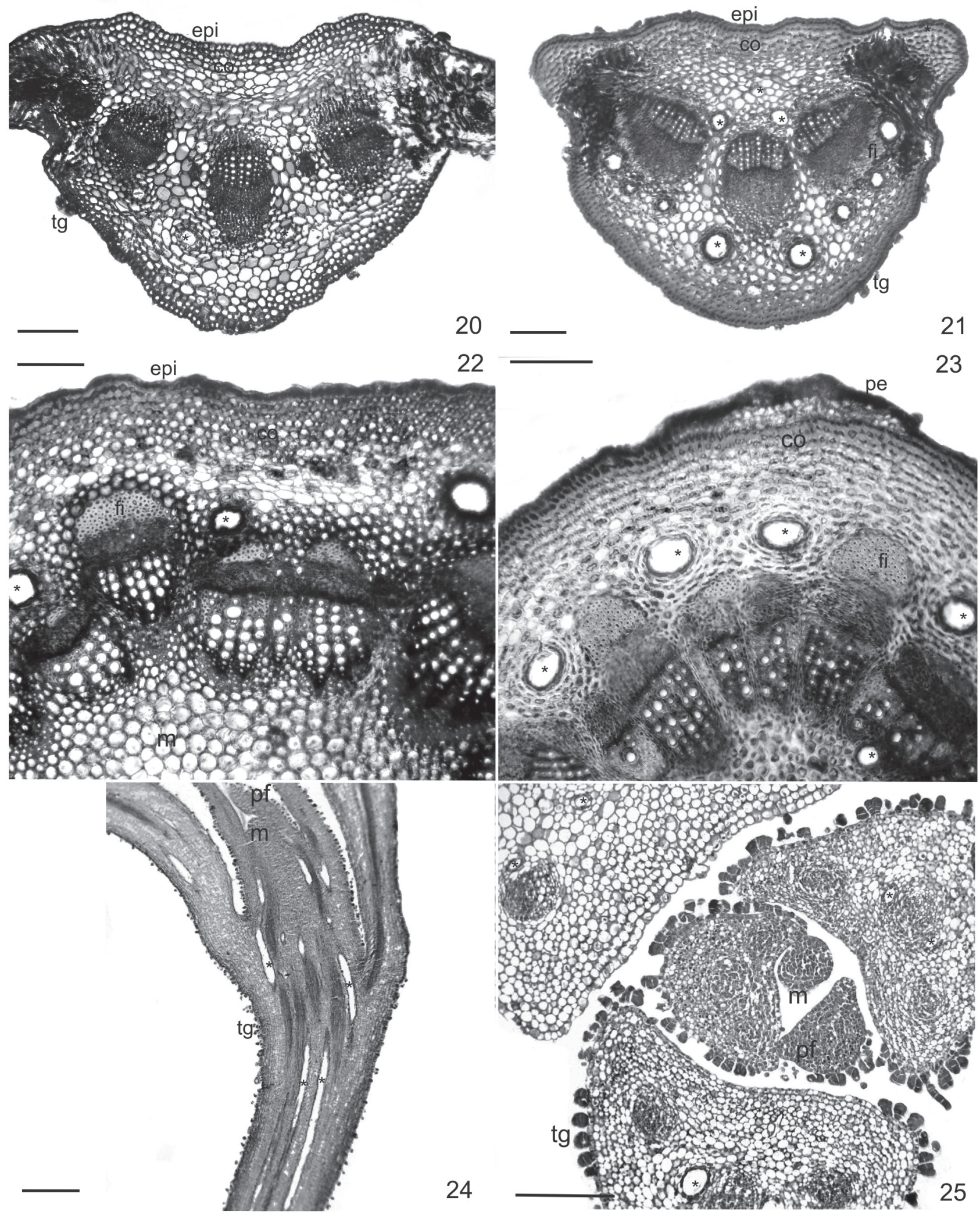

Figura 20-25. Corte transversal por pecíolo de F. hirta y F. leptopoda. 22, corte transversal por tallo primario de $F$. hirta. 23, comienzo de crecimiento secundario de F. tortuosa. 24 y 25. Corte longitudinal y transversal de apice caulinar de F. niederleinii. Escala: $20-21=200 \mu \mathrm{m}$.; $22-23=150 \mu \mathrm{m} .24=500 \mu \mathrm{m}$. $25=100 \mu \mathrm{m}$. Abreviaturas: co: colenquima, epi: epidermis, fi: fibras, m: meristema apical, pe: peridermis, pf: primordio foliar, ${ }^{*}$ conductos secretores. 
Tabla 1. Características cuantitativas foliares en especies de Flourensia DC. (Asteraceae). Las mediciones se expresan en mm y el área en mm ${ }^{2}$. Los resultados se muestran como media \pm desvío estándar. Test de Tukey, diferentes letras indican diferencias significativas.

\begin{tabular}{|c|c|c|c|c|c|c|c|c|}
\hline & $\begin{array}{c}\text { Lámina } \\
\text { largo }\end{array}$ & $\begin{array}{c}\text { Lámina } \\
\text { ancho }\end{array}$ & $\begin{array}{l}\text { Lámina } \\
\text { espesor }\end{array}$ & $\begin{array}{c}\text { Vena } \\
\text { espesor }\end{array}$ & $\begin{array}{c}\text { Pecíolo } \\
\text { largo }\end{array}$ & $\begin{array}{l}\text { Pecíolo } \\
\text { ancho }\end{array}$ & $\begin{array}{l}\text { Pecíolo } \\
\text { espesor }\end{array}$ & Área foliar \\
\hline F. hirta & $\begin{array}{l}49,75 \pm \\
9,24 \text { a }\end{array}$ & $\begin{array}{c}7,1 \pm \\
1,07 \text { a }\end{array}$ & $\begin{array}{c}0,32 \pm \\
0,06 \mathrm{ab}\end{array}$ & $\begin{array}{c}0,55 \pm \\
0,09 \quad \text { a }\end{array}$ & $\begin{array}{c}2,33 \pm \\
0,61 \quad a\end{array}$ & $\begin{array}{l}1,15 \pm \\
0,22 \quad a\end{array}$ & $\begin{array}{c}0,61 \pm \\
0,13 \text { a }\end{array}$ & $\begin{array}{c}258,04 \pm \\
74,03 \mathrm{a}\end{array}$ \\
\hline F. leptopoda & $\begin{array}{l}44,55 \pm \\
6,92 \text { a }\end{array}$ & $\begin{array}{l}22,9 \pm \\
2,85 \mathrm{c}\end{array}$ & $\begin{array}{c}0,28 \pm \\
0,06 \text { a }\end{array}$ & $\begin{array}{l}0,65 \pm \\
0,12 \quad b\end{array}$ & $\begin{array}{c}8,8 \pm \\
2,21 \quad \mathrm{c}\end{array}$ & $\begin{array}{l}1,33 \pm \\
0,13 \text { a }\end{array}$ & $\begin{array}{c}0,93 \pm \\
0,15 \quad b\end{array}$ & $\begin{array}{l}659,37 \pm \\
178,67 \mathrm{~b}\end{array}$ \\
\hline F. niederleinii & $\begin{array}{c}63,15 \pm \\
13,16 \quad b\end{array}$ & $\begin{array}{l}11,65 \pm \\
2,01 \quad b\end{array}$ & $\begin{array}{c}0,32 \pm \\
0,09 \mathrm{ab}\end{array}$ & $\begin{array}{c}0,61 \pm \\
0,11 \mathrm{ab}\end{array}$ & $\begin{array}{r}6,5 \pm \\
2,28 \quad b\end{array}$ & $\begin{array}{c}1,18 \pm \\
0,22 \quad a\end{array}$ & $\begin{array}{c}0,78 \pm \\
0,19 \quad b\end{array}$ & $\begin{array}{l}541,58 \pm \\
205,01 \text { b }\end{array}$ \\
\hline F. tortuosa & $\begin{array}{l}75,45 \pm \\
12,36 \mathrm{c}\end{array}$ & $\begin{array}{l}25,05 \pm \\
4,86 \text { c }\end{array}$ & $\begin{array}{c}0,38 \pm \\
0,09 \quad b\end{array}$ & $\begin{array}{l}0,89 \pm \\
0,1 \quad \mathrm{c}\end{array}$ & $\begin{array}{c}9,2 \pm \\
2,69 \mathrm{c}\end{array}$ & $\begin{array}{c}2,03 \pm \\
0,35 \quad b\end{array}$ & $\begin{array}{l}1,38 \pm \\
0,2 \quad \mathrm{c}\end{array}$ & $\begin{array}{c}1385,3 \pm \\
80,66 \mathrm{c}\end{array}$ \\
\hline
\end{tabular}

Tabla 2. Características cuantitativas epidérmicas para cada epidermis foliar de especies de Flourensia DC. (Asteraceae). Las variables de frecuencias estomática y de células epidérmicas propiamente dichas se expresan como media \pm desvío estándar, IE $=$ índice estomático. Anova con $\mathrm{p} \leq 0,05 \mathrm{y} 1$ grado de libertad, ${ }^{*}=$ diferencias significativas entre las caras de la misma especie.

\begin{tabular}{|c|c|c|c|c|}
\hline Especie & Cara foliar & Frec. Estomática & Frec. Cel. Epid. & IE \\
\hline F. hirta & $\begin{array}{l}\text { Hipofilo } \\
\text { Epifilo }\end{array}$ & $\begin{array}{c}123,68 \pm 25,85 \\
115,35 \pm 18,7\end{array}$ & $\begin{array}{c}845,83 \pm 164,13 \\
937,5 \pm 190,68\end{array}$ & $\begin{array}{l}12,76 \\
10,96\end{array}$ \\
\hline F. leptopoda & $\begin{array}{l}\text { Hipofilo } \\
\text { Epifilo }\end{array}$ & $\begin{array}{c}182,29 \pm 53,2 \\
149,02 \pm 33,15\end{array}$ * & $\begin{array}{l}1083,33 \pm 318,51 \\
1145,83 \pm 317,57\end{array}$ & $\begin{array}{c}14,4 \\
11,51\end{array}$ \\
\hline F. niederleinii & $\begin{array}{l}\text { Hipofilo } \\
\text { Epifilo }\end{array}$ & $\begin{array}{c}169,34 \pm 31,77 \\
124,45 \pm 31,49\end{array}$ * & $\begin{array}{l}1141,98 \pm 186,52 \\
1198,56 \pm 190,84\end{array}$ & $\begin{array}{c}12,91 \\
9,41\end{array}$ \\
\hline F. tortuosa & $\begin{array}{l}\text { Hipofilo } \\
\text { Epifilo }\end{array}$ & $\begin{array}{c}135,86 \pm 27,46 \\
81,39 \pm 39,66\end{array}$ * & $\begin{array}{l}1433,33 \pm 205,72 \\
1300 \pm 164,18\end{array}$ * & $\begin{array}{l}8,66 \\
5,89\end{array}$ \\
\hline
\end{tabular}

Tabla 3. Test de Tuckey, comparación entre los hipofilos y epifilos de especies de Flourensia DC. (Asteraceae) para la variable de frecuencia estomática. Se incluyen las medias para su mejor visualización. Diferentes letras representan diferencias significativas.

\begin{tabular}{|c|c|c|c|c|c|c|c|}
\hline & \multicolumn{3}{|c|}{ HIPOFILO } & \multicolumn{4}{|c|}{ EPIFILO } \\
\hline Especie & Medias & & & Medias & & & \\
\hline F. tortuosa & 135,86 & A & & 81,39 & A & & \\
\hline F. hirta & 123,68 & A & & 115,35 & & B & \\
\hline F. niederleinii & 169,34 & & B & 124,45 & & B & \\
\hline F. leptopoda & 182,29 & & B & 149,02 & & & $\mathrm{C}$ \\
\hline
\end{tabular}

y patógenos, ya que sólo saldrían al exterior si la planta es herida, además de contribuir al balance hídrico (Fahn 1979).

La presencia de estas estructuras secretoras permite predecir que las especies de Flourensia estudiadas en el presente trabajo, también tendrían gran cantidad de compuestos químicos con valor potencial, por lo que entendemos que es necesario ampliar las investigaciones fitoquímicas para confirmarlo.

\section{Agradecimientos}

Agradecemos al Dr. Luís Ariza Espinar por la determinación del material, a la Dra. Nilda Dottori por sus sugerencias y a los revisores de la revista por sus correcciones. Agradecemos a la Secretaría de Ciencia y Técnica de la Universidad
Nacional de Córdoba, Argentina, al por el apoyo económico brindado. El presente trabajo es parte de la tesis doctoral de la primera autora, la cual cursa la carrera de Doctorado en Ciencias Biológicas de la Universidad Nacional de Córdoba, Argentina, y es becaria del Consejo Nacional de Investigaciones Científicas y Técnicas (CONICET).

\section{Referencias}

Aguilera, D.; Meira, R.; \& Ferreira, F. 2004. Anatomía e histoquímica dos órgãos vegetativos de Siegesbeckia orientalis (Asteraceae). Planta daninha, Viçosa, Minas Gerais 22(4): 483-489.

Ariza Espinar, L. 2000. Familia Asteraceae. Tribu Heliantheae. Pródromo de la Flora Fanerogámica de Argentina Central. 2: 1-111. Museo Botánico. Fac. de C. E. F. y N. (UNC). Córdoba, Argentina.

Askew A. 2003. Leaf Area Measurement. Version 1.3. The University of Sheffield. UK.

Barbosa, G.; Cantero, J.; Nunez, C. \& Ariza Espinar, L. 2006. Flora medicinal de la provincia de Córdoba (Argentina). Museo Botánico Córdoba.

Bohlmann, F. \& Jakupovic J. 1979. Neue Sesquiterpene, Triterpene, Flavanone und andere aromatische Verbindungen aus Flourensia heterolepis. Phytochemistry 18 (7): 1189-1194.

Budel, J. \& Duarte, M. 2007. Caracteres Morfoanatômicos de Partes Vegetativas Aéreas de Baccharis coridifolia DC. (Asteraceae-Astereae). Latin American Journal of Pharmacy 26(5): 723-31.

Carpinella, M.; Ruiz, G. \& Palacios, S. 2010. Screening of native plants of central Argentina for antifungal activity. Allelopathy Journal 25(2): 423-431.

Carrizo Flores, R.; Vaca, L.; Donade, O.; Laciar, A. \& Tonn, C. 2005. Antibacterial effect of Baccharis salicifolia and Flourensia oolepis essential oils on Listeria monocytogenes. Biocell 29 (3): 357-366. 
Castro, M.; Leitao-Filho, H.; \& Monteiro, W. 1997. Utilização de estruturas secretoras na identificação dos gêneros de Asteraceae de uma vegetação de cerrado. Revista Brasileira de Botanica 20 (2): 163-174.

D’ Ambrogio de Argüeso, A. 1986. Manual de Técnicas de Histología Vegetal. Pp. 1-83 Ed. Hemisferio Sur. Argentina.

Delbón, N.; Cosa, M. T. \& Dottori, N. 2007a. Anatomía de órganos vegetativos en Flourensia campestris y F. oolepis (Asteraceae), con especial referencia a las estructuras secretoras. Arnaldoa 14(1): 61-70.

Delbón, N.; Cosa, M. T.; Dottori, N. \& Stiefkens, L. 2007b. Estudio de la epidermis foliar en Flourensia campestris y F. oolepis (Asteraceae). Boletín de la Sociedad Argentina de Botánica 42(3-4): 245-250.

Di Rienzo, J.; Casanoves, F.; Balzarini, M.; Gonzalez, L.; Tablada, M.; Robledo, C. InfoStat, versión 2009. Grupo InfoStat, F. C. A (UNC). Córdoba, Argentina.

Diaz Napal, G.; Carpinella, M. \& Palacios, S. 2009. Antifeedant activity of ethanolic extract from Flourensia oolepis and isolation of pinocembrin as its active principle. Bioresource Technology 100: 3669-3673.

Dickison, W.C. 2000. Integrative Plant Anatomy. Academic Press. U.S.A.

Dillon, M.O. 1984. Systematic study of Flourensia. Fideldiana Botany 16: 1-67.

Donadel, O.; Guerreiro, E.; Wendel, M.; Enriz, G.; Guiordano, R. \& Tonn, C. 2005. Gastric cytoprotective activity of ilicic aldehyde: Structureactivity relationships. Bioorganic \& Medicinal Chemistry Letters 15: 547-550.

Estell, R.; Fredickson, E. \& Havstad, K. 1996. Chemical composition of Flourensia cernua al four growth stages. Grass and Forage Science 15: 434-411.

Evert, R. F. 2006. Esau's Plant anatomy. 447-473. John Wiley \& Sons, Inc., Hoboken, New Jersey.

Fahn, A. \& Cutler, D. 1992. Xerophytes. 1-143 Handbuchder Pflanzenantomie XIII, 3. Gebruder Borntraeger, Berlín.

Fahn, A. 1979. Secretory tissues in plants. Academic Press. London.

Fahn, A. 1990. Plant Anatomy. Pergamon Press. Oxford.

Funk, A.; Susanna, A.; Stuessy, T. \& Robinson, H. 2009. Classification of Compositae. Systematics, evolution, and biogeography of Compositae. Michigan, USA. Sheridan Books Inc.

Gamboa-Alvarado, R.; Hernandez-Castillo, F.; Gerrero-Rodrigues, E. \& Sanchez-Arizpe, A. 2003. Inhibición del crecimiento micelial de Rhizoctonia solani Kuhn. y Phytophthera infestant Mont. (De Bary) con extractos vegetales metanólicos de Hojasen (Flourensia cernua DC.) Mejorana (Origanum mejorana L.) y Trompetilla (Bouvardia ternifolia (Ca.) Schelecht. Revista Mexicana de Fitopatología 21: 13-18.

García, M.; Gonzalez-Coloma, A.; Donadel, O.; Ardanaz, C.; Tonn, T. \& Sosa, M. 2007. Insecticidal effects of Flourensia oolepis Blake (Asteraceae) essential oil. Biochemical Systematics and Ecology 35(4): 181-187.

Gerrero-Rodriguez, E.; Solis-Gaona, S.; Hernandes-Castillo, F.; FloresOlivas, A. \& Sandoval-Lopez, V. 2007. Actividad biológica in vitro de extractos de Flourensia cernua DC. en patógenos de postcosecha: Alternaria alternata (Fr.:Fr.) Keissl., Colletotrichum gloeosporioides (Penz.) Penz. y Sacc. y Penicillium digitatum (Pers.:Fr.) Sacc. Revista Mexicana de Fitopatología 25: 48-53.

Jasso de Rodriguez, D.; Hernandez-Castillo, D.; Angulo-Sanchez, J.; Rodrıguez-Garcıa, R.; Villarreal Quintanilla, J. \& Lira-Saldivar, R. H. 2007. Antifungal activity in vitro of Flourensia spp. extracts on Alternaria sp., Rhizoctonia solani, and Fusarium oxysporum. Industrial Crops and Products 25: 111-116.

Joray, M.; del Rollán, M.; Ruiz, G.; Palacios, S. \& Carpinella, M. 2011. Antibacterial Activity of Extracts from Plants of Central ArgentinaIsolation of an Active Principle from Achyrocline satureioides. Planta Medica 77: 95-100.

Kraus, J.; de Sousa, H.; Rezende, M.; Castro, N.; Vecchi, C. \& Luque, R. 1998. Astra Blue and Basic Fuchsin double staining of plant materials. Biotechnic \& Histochemistry 73(5): 235-243.

Lapp, M.; Jáuregui, D. \& Ruiz-Zapata, T. 2004. Anatomía foliar de ocho especies venezolanas del género Oyedaea DC. (AsteraceaeHeliantheae). Acta Botánica Venezuelica 27(1): 1-16.
Luti, R. 1979 en Vázquez, J.; Miatello, R. \& Roqué, M. 1979. Geografía física de la provincia de Córdoba. 297-368 Ed. Boldt. Argentina.

Mata, R.; Bye, R.; Linares, E.; Macias, M.; Rivero-Cruz, I.; Perez, O \& Timmermann, B. 2003. Phytotoxic compounds from Flourensia cernua. Phytochemistry 64: 285-291.

Melo-De-Pinna, G. \& Menezes, N. 2002. Vegetative organ anatomy of Ianthopappus corymbosus Roque \& Hind (Asteraceae-Mutisieae) Revista Brasileira de Botânica 25(4): 505-514.

Molares, S.; González, S.; Ladio, A \& Agueda Castro, M. 2009. Etnobotánica, anatomía y caracterización físico-química del aceite esencial de Baccharis obovata Hook. et Arn. (Asteraceae: Astereae) Acta Botanica Brasilica 23(2): 578-589.

Molina-Salinas, G.; Ramos-Guerra, M.; Vargas-Villarreal, J.; Mata-Cárdenas, B.; Becerril-Montes, P. \& Said-Fernández, S. 2006. Bactericidal Activity of Organic Extracts from Flourensia cernua DC against Strains of Mycobacterium tuberculosis. Archives of Medical Research 37(1): 45-49.

Monteiro, W.; Castro, M.; Mazzoni-Viveiros, S. \& Mahlberg, P. G. 2001. Development and some histochemical aspects of foliar glandular trichomes of Stevia rebaudiana (Bert.) Bert. Asteraceae. Revista Brasileira de Botânica 24 (3): 349-357.

Pacciaroni, A.; Arjona, M.; Ariza Espinar, L. \& Sosa, V.E. 2009. Benzofuranos y eudesmanólidos de Flourensia blakeana Dillon. Anais... XVII Simposio Nacional de Química Orgánica. Mendoza, Argentina. Res.

Priotti, Z.; Zygadlo, J. \& Ariza Espinar, L. 1997. Essential oils of Flourensia oolepis S. L. Blake. Journal of Essential Oil Research 9: 345-347.

Ramayya N. 1962. Studies on de trichomes of some Compositae I. General structure. Bulletin of the Botanical Survey of India 1-4: 177-188.

Rao, M.; Kingston, D. \& Spittler, T. Flavonoids from flourensia cernua. 1970. Phytochemistry 9(1): 227-228.

Sáenz, A. 2000. en F. O. Zuloaga \& O. Morrone (Eds.). Catálogo de las plantas vasculares de la República Argentina. II Asteraceae 90-91. Monographs in Systematic Botany fom the Missouri Botanical Garden.

Scheffe, H. 1959. The Analisis of Variance. New York, Ed. John Wiley.

Simon, P.; Katinas, L. \& Arambarri, A. 2002. Secretory structures in Tagetes minuta (Asteraceae, Helianieae). Boletín de la Sociedad Argentina de Botánica 37(3-4): 181-191.

Sokal, R. \& Rohlf, F. 1981. The Principles and Pratice of Statistic in Biological Research. Ed. Freeman and Co. San Francisco.

Stace, C.A. 1965. Cuticular studies as an aid to plant taxonomy. Bulletin of British Musseum Natural History 4: 62-63.

Telles, M.; Estell, R.; Fredickson, E. \& Havstad, K. 1997. Essential oil in Flourensia cernua DC. Journal of Essential Oil Research 9: 619-624.

Telles, M.; Estell, R.; Fredickson, E.; Powell, J.; Wedge, D.; SCHRADER, K. \& Kobaisy, M. 2001. Extracts of Flourensia cernua (L): Volatile constituents and antifungal, antialgal, and antitermite bioactivities. Journal of Chemical Ecology 27 (11): 2264-2273.

Trillo, C.; Demaio, P.; Colantonio, S. \& Galetto, L. 2007. Conocimiento actual de plantas tintóreas por los pobladores del valle de Guasapampa, provincia de Córdoba. Kurtziana 33(1): 65-71.

Uriburu, M.; De la Fuente, J.; Palermo, J.; Gil, R. \& Sosa, V. 2004. Constituents of two Flourensia species. Phytochemistry 65(14): 2039-2043.

Uriburu, M.; de la Fuente, J.; Palermo, J. \& Sosa, V. 2005. A Chlorinated dihydrobenzofuran from Flourensia riparia. Journal of the Argentine Chemical Society 93: 161-164.

Uriburu, M.; Gil, R.; Sosa, V. \& de la Fuente, J. 2007. Prenylflavonoids from Flourensia fiebrigii. Phytochemistry 68: 1295-1299.

Vaca Ruiz, M.; Laciar, A.; Donadel, O.; Saad, J. \& Carrizo Flores, R. 2006. Anti-listerial activity of plant essential oils from western region of Argentina. Annals of Microbiology 56(4): 369-371.

Zardini, E. 1984. Etnobotánica de Compuestas Argentinas con especial referencia a su uso farmacológico. Acta Farmacéutica Bonaerense 3(1): 77-99.

Zavala, D.; Carrillo, M.; Alvarado, B.; Sánchez, A. 2010 Evaluación de la toxicidad aguda de un extracto alcohólico de hojas de hojasén (Flourensia cernua). Revista Mexicana de Ciencias Farmacéuticas 41 (3): 50-54. 